Background. Diagnosing asthma in pre-school children with asthma-like symptoms is a major problem. From a clinical viewpoint, because of the specificity and variability of symptoms, it is currently almost impossible to distinguish the asthmatic from the non-asthmatic child. Since asthma is defined as a reversible airway obstruction reversibility testing could be of additional value.

Aim. To assess reversibility of lung function in pre-school children with asthma or asthma-like symptoms. Methods. In symptomatic children, included in the ASTERISK (Astma, Symptomen, Therapie En RISiKo factoren) study, airway resistance (pre/post salbutamol) was measured by means of interrupter technique (Rint) and forced oscillation technique (FOT) when possible.

Results. 96 Children were included in the ASTERISK study (69\% male; mean age 2.6 (1.2) yr.). Lung function measurements were successful in $53(55 \%)$ and 35 (36\%) children (respectively Rint and FOT). The succes rate increases with age (table 1). Lung function significantly improved after salbutamol (table 2).

Table 1. Success rates (\%): 5 acceptable measurements.

Age

$1-2$ yr $(\mathrm{n}=38)$

$2-3 \mathrm{yr}(\mathrm{n}=21)$

$3-4 \mathrm{yr}(\mathrm{n}=19)$

$4-5$ yr $(n=18)$

$\begin{array}{rr}\text { Rint } & \text { FOT } \\ 16 \% & 0 \% \\ 71 \% & 38 \% \\ 74 \% & 53 \% \\ 100 \% & 94 \%\end{array}$

Table 2. Lung function pre/post salbutamol (mean (S.D.));

\section{Lung function}

Rint $(\mathrm{n}=53)$

FOT $(\mathrm{n}=35)$

Resistance (kPa.s/L)

Pre

Resistance (hPa.s/L) $7.8(1.7)$

Post

Resistance at $6 \mathrm{~Hz}($

\section{${ }^{*} \mathbf{p}<0.05$ after Wilcoxon $\mathbf{U}$ test.}

Conclusion. Reversibility testing in pre-school children with asthma or asthma-like symptoms is possible using Rint and FOT. However, the diagnostic value of this measurement needs to be determined further.

Co-authors: S. Schokker, E M W Kooi, P L P Brand, T W de Vries, E J Duiverman

Funded by GlaxoSmithKline

A focus group study on the impact of the different components of pulmonary rehabilitation

AB12PO

Rupert C M Jones, Research Unit, Peninsula Medical School, Plymouth

Prim Care Resp J 2003; 12(2):65

Background: Multi-professional pulmonary rehabilitation (PR) has been shown to improve exercise tolerance and quality of life. It is not known which aspects of PR (e.g. exercise versus education versus social support) are found to be most helpful by patients and existing quality of life tools do not explore this issue.

Methods: Six focus groups were held three months after PR with patients recruited from two programmes. One being a typical intensive, hospital-based scheme (Torquay) and the other, a short, once weekly programme based in various locations in the community (Plymouth).

Results: Perceived effects of education included reduced fear of dyspnoea, improved use of benefit system and improved drug compliance. Perceived effects of social context included encouragement during exercise and smoking cessation, and new social activities amongst group members. Exercise in a safe environment increased confidence in activity and also reduced fear of dyspnoea, leading to new activities (e.g. holidays, shopping trips, etc). Patients judged PR to be more helpful than medical interventions. There appeared to be more extra-curricula social contact in the community group.

Conclusions: Patient reported benefits of PR can be attributed to exercise, education and social context, supporting the use of multiprofessional, multi-component PR programmes. Peer group support in both programmes appear to be an important factor in behavioral change.

Co-authors: M Gray, M Hyland and J Goldman

Source of funding: Boehringer-Ingelheim $£ 3,000$

An Assessment of a mobile COPD specialist nurse service in primary care

AB13PR

Rupert C M Jones, Research Unit, Peninsula Medical School, Plymouth

Prim Care Resp J 2003; 12(2):65-66

Background: COPD is a growing burden on the NHS which is preventable if diagnosed early and combined with smoking cessation. Diagnosis requires spirometry. A recent survey demonstrated major problems with existing spirometry in primary care. Many practices have no spirometer or have problems with interpreting the results. This study assessed the value of a trained Respiratory Specialist Nurse (RSN) offering structured COPD diagnostic and management services to practices in Plymouth PCT.

Methods: A package of services was offered to a random sample of practices in Plymouth by the RSN. All these practices owned a spirometer. The package included:

- Education for practice staff on spirometry technique, interpretation of data and COPD management.

- Spirometry clinics performed by the RSN on patients referred with dyspnoea, either at the surgery or a nearby community clinic.

Assessments included bronchodilator and steroid reversibility testing. Clinics were performed with or without the practice nurse being present.

- Spirometry results were analysed by the RSN and a GP with special interest in respiratory disease. Diagnosis and suggestions for ongoing 
management were then fed back to the practices.

- Guidance on appropriate referrals to other COPD services available locally, e.g. smoking cessation, pulmonary rehabilitation, physiotherapy and respiratory specialist nurses.

Results: The service was offered to 14 practices of which 7 declined. In the 7 participating practices, 98 patients with breathing problems were assessed by the RSN over 3 months: 19 had asthma; 68 COPD (mild 25, moderate 16, severe 27); 5 had a pure restrictive picture; and 6 were normal. Only 10 patients had a previous diagnosis of COPD. High levels of satisfaction were reported by practices and patients. 22 patients were current cigarette smokers; 65 requested information about their disease; 52 had poor inhaler technique; 25 needed consultant referral; 20 needed referral to pulmonary rehabilitation.

Conclusions: In practices in Plymouth that owned a spirometer there was often a lack of structured approach, skills and confidence to deal with breathless patients. This mobile COPD service diagnosed many new cases of COPD but often at a late stage. Once diagnosed, appropriate management can be started to control symptoms and reduce disease progression. Strategies included drug and oxygen therapy, consultant and specialist nurse support, pulmonary rehabilitation and smoking cessation. The advice about COPD management was well received. Currently, early diagnosis which can prevent COPD progression is not happening - this service could save lives.

Co-authors: Whittaker M, Hanney KE

Funding: Glaxo $£ 3000$, Boehringer Ingelheim £2000, Plymouth PCT $£ 2000$

Complexity in patient experience of Chronic Obstructive Pulmonary Disease: a qualitative study

Rachel L Parayil, for Health Services Research, School of Population \& Health Sciences, University of Newcastle

AB14PO

Prim Care Resp J 2003; 12(2):66

Rationale: Exacerbations of chronic obstructive pulmonary disease (COPD) are an important health outcome with significant relationship to quality of life, but little is known about patient perspectives of COPD \& its exacerbations.

Methods: We examined patient attitudes toward, \& experiences of their disease and its exacerbations. A convenience sample of 14 patients from Gateshead, Tyne \& Wear, UK, who had seen a health care professional (HCP) regarding their COPD in the last 6 weeks were recruited for in-depth, tape recorded interviews \& spirometry. 11 met the criteria and had interviews recorded. Data were analysed for notable themes and their patterns.

Results: The results found a high level of variation (not correlated with lung function) with few shared patterns of experience. The results listed here then seek to describe the patients' experiences and their many varied nature.

- Breathing problems might have begun 'as a kid,' during one's 40 s or one's $60 \mathrm{~s}$.

- For one participant life just now was like having been let out of prison (since commencing 'Slo-Phyllin'), but another sits crying and wondering if 'is it worth bothering?'

- Social activities could constitute 4 trips to the day centre a week or not getting out at all.

- One participant maintains a social function - doing the school run, whilst another 'just sits.'

- The disease can be 'alright one day, then all around the next,' vary on a week to week basis, or not at all.

- In terms of day to day symptoms, the biggest barrier caused by the disease might not be breathlessness, it could be tiredness or cough the struggle to expectorate causing the breathlessness or there might be no cough. It could be a mainly nocturnal problem, no bother at all at night, or worst in the mornings.

- This can be a disease with no pain, or one where the struggle to breathe makes you ache.

- The dry heat of foreign climes could be sought after, or the dense summer air feared.

- One man 'loses his breath' in the cold Tyneside air, but not in bitterly cold, snowy Hamburg.

- Descriptions of how exacerbations start showed that there could be a sudden, frightening inability to breathe, simply a change in phlegm colour, or something identified during a check up for something else.

- Treatment could mean being rushed into hospital, being given a script, or no treatment at all.

- Exacerbations might be resolved within days of commencing medication, or unresolved 6 months later.

- Oral steroids could be wonder drugs, 'I felt great,' or be completely ineffective.

Conclusions: The above may come as no surprise, but how does it influence practice? A patient centred approach is advocated for doctorpatient consultations today. The study described here is an example as to importance of such an approach. It paints a picture of a many varied disease which poses the question, 'Does this fit with HCP perceptions of COPD?'

Co-authors: K Jones, D Price \& D Nonikov

Funded by: Boehringer Ingelheim 